\title{
Effects and Ways of Tailored Gamification in Software-Based Training in Cognitive Rehabilitation
}

\author{
Mareike Gabele \\ mareike@isg.cs.uni-magdeburg.de \\ Otto von Guericke University \\ Magdeburg \\ Magdeburg, Germany \\ Andrea Thoms \\ HASOMED GmbH \\ Magdeburg, Germany
}

\author{
Juliane Weicker \\ Clinic of Cognitive Neurology, \\ University of Leipzig \\ Leipzig, Germany \\ weicker@cbs.mpg.de \\ Steffi Hußlein \\ Magdeburg-Stendal University of \\ Applied Sciences \\ Magdeburg, Germany
}

\author{
Sebastian Wagner \\ Otto von Guericke University \\ Magdeburg \\ Magdeburg, Germany
}

\author{
Christian Hansen \\ Otto von Guericke University \\ Magdeburg \\ Magdeburg, Germany
}

\begin{abstract}
A high level of motivation and frequent training are relevant in software-based rehabilitation to improve cognitive functioning after acquired brain injury. We evaluated the benefit of tailored usercentered gamification elements in a clinical study with $\mathrm{N}=83$ outpatients undergoing three weeks of cognitive training in their home environment. The use of gamification in relation to the patient's player type was explored in three steps. First, we determined the individual player types and related requests for specific game elements by means of questionnaires. Afterwards, we examined the effect of gamified training based on a non-player character and training progress within a metaphor. We considered secondly the individual perception and emotional effect and thirdly the performance based on training duration. 37 elements were requested by patients of all types, 18 elements were partially requested, and 4 elements were rejected. A comparison shows that the requested game elements partly differ between healthy persons and patients. Overall, gamification was perceived positively and gamified training leads to an increase in enjoyment compared to non-gamified training. In detail, however, there were different effects on the individual player types: socialisers experienced more enjoyment while achievers perceived higher competence throughout gamified cognitive training. Also, differences in performance in training duration were found. Within gamified training, socialisers trained significantly more than patients not primarily assigned to this type. In contrast, no significant difference was found for achievers. By showing modulating requests and effects in player types, our results support user-centered tailoring of game elements in the development of software-based cognitive training in rehabilitation.
\end{abstract}

\section{CCS CONCEPTS}

- Human-centered computing $\rightarrow$ User studies.

Permission to make digital or hard copies of part or all of this work for personal or classroom use is granted without fee provided that copies are not made or distributed for profit or commercial advantage and that copies bear this notice and the full citation on the first page. Copyrights for third-party components of this work must be honored

For all other uses, contact the owner/author(s).

UMAP '21, June 21-25, 2021, Utrecht, Netherlands

(C) 2021 Copyright held by the owner/author(s).

ACM ISBN 978-1-4503-8366-0/21/06.

https://doi.org/10.1145/3450613.3456828

\section{KEYWORDS}

Gamification, Rehabilitation, Motivation

ACM Reference Format:

Mareike Gabele, Juliane Weicker, Sebastian Wagner, Andrea Thoms, Steffi Hußlein, and Christian Hansen. 2021. Effects and Ways of Tailored Gamification in Software-Based Training in Cognitive Rehabilitation. In Proceedings of the 29th ACM Conference on User Modeling, Adaptation and Personalization (UMAP '21), fune 21-25, 2021, Utrecht, Netherlands. ACM, New York, NY, USA, 11 pages. https://doi.org/10.1145/3450613.3456828

\section{INTRODUCTION}

Acquired cognitive impairment in adulthood results in most cases from strokes [42]. Functionally effective software-based training is used in cognitive rehabilitation after acquired brain injury [68, 70]. In addition to therapy in clinics, supplementary home training can be conducted. A higher number of training sessions has a positive impact on its effectiveness [67]. However, longer training durations can lead to motivation problems. Insufficient training or too early dropping out is a problem in e-Health [14]. The overall exocentric goal [9] in software-based training in cognitive rehabilitation is to use relearned abilities in daily life. To relearn them, the goal is initially endocentric to use the training software, continue training and promote positive emotions.

\subsection{Tailoring Gamification in Health}

In health, gamification is used in software to support motivation and behavioral change [2, 26, 49]. Gamification describes the use of game elements in a non-game context [11], such as rehabilitation. Thereby gamification should be understood as a process, whose goal is to support value creation for the user through gameful experience [24]. Mechanics, like specifying goals and clarifying progress, but also emerging emotions like fun, are an essential basis for this [54]. In health, most research results on gamification range from mixed to positive [35]. Most commonly used elements in ehealth are rewards and feedback [59]. Gamification may lead to an increase in motivation, but this cannot be generalised for every element and user. For example, adverse effects have been observed in leaderboards which may lead to reduced feelings of competence or social relatedness [6]. However, perceived competence (learn 
useful skills), social relatedness (feel belonging to others) and autonomy (determine your behavior) is the basis for developing intrinsic motivation according to the self-determination theory [56]. Game Elements are perceived differently by users. Based on their character, users can be assigned to different player types [3, 4, 46, 69]. 'Player and User Types Hexad' differentiates between six types motivated by different aspects: philanthropist (altruism), free spirit (autonomy), achiever (competence), socialiser (social relatedness), player (rewards), and disruptor (change) $[3,65]$. The preferences for specific game elements correlates with the player types [63, 65]. Tailored elements can have a positive impact on perception, task performance and user engagement [47, 64]. In health related behavioral changes, tailored game elements depending on the players' character are ways to influence the effectiveness of training [47] or healthy eating $[29,48]$. It is important to stimulate positive effects while avoiding negative effects $[29,48]$, e.g. to overwhelm the user by introducing too many strategies [34], especially for clinical use. It is highly relevant to select specific elements suitable for the user to achieve the desired effects and compliance [31]. Therefore, knowing the target group, their characteristics, needs and behavior are essential for most gamification frameworks [44, 45]. In this vein, the context in which gamification is used is relevant for its effect on user motivation [20,22]. Previous studies point out that there may be differences in motivation and performance of patients with brain injury compared to healthy persons [7]. However, to realize a user-centred approach for this target group, the request for game elements in cognitive rehabilitation needs to be further investigated. This leads us to Research Question (RQ) 1: Which game elements are requested by patients in software-based training in cognitive rehabilitation depending on their player type?

\subsection{Emotions and Behavior in Training}

Essential for the drive to perform are the mechanics of visual and positive feedback [10]. In sports, health programs and apps, usually the user's progress is visualized. Feedback on progress shows success in achieving the goal and can support repetition of a behavior [54]. User performance increases significantly by implementing the combination of progress bar and feedback [41]. In therapy, reporting training progress to patients and feedback on recovery are essential factors for patients and improve performance [23, 32]. According to goalsetting theory, the behavior is related to goals and the feedback given for them [13,39]. Based on the received feedback behavior can be regulated accordingly to foster progress towards this goal [28]. Research in gamification elements showed that progression is among others related to the gamification element's meaning or purpose [63]. In current cognitive rehabilitation trainings for adults, progress is often visualized in classic charts, such as lines [52] or bars [19]. They represent past progress but provide a limited basis for future steps and a less meaningful user-oriented integration of the overall goal. Based on serious games, a case study suggests that seeing one's improvements may increase the motivation for training [1]. Progress visualizations that enable to track one's improvements have been successfully realized by a metaphor (using a map or a path) and avatars or Non-Player Character (NPCs), who walk along such paths to illustrate the progress. Likewise, digital characters show the possibility of supporting behavioral changes through feedback [38]. Social reinforcement can arise through real or digital contacts with other people, but also through non-real contacts with digital team members such as NPCs [57]. Brain injury can lead to limitations in social cognitive functions, which can make it difficult for people to integrate socially [33]. However, social isolation and lack of social support can lead to poorer rehabilitation outcomes [17] and higher mortality [55]. Strengthened social relatedness leads to feeling more involved [5]. It has been shown that older adults benefit from presenting a virtual character in comparison to text or speech [51]. For example, instructions could be better followed and contact was perceived as pleasant. Adverse effects on memory or recognition of emotions were not reported. However, it is unclear how an NPC and progression metaphors are perceived in clinical conditions when users suffer from cognitive impairments. This leads us to Research Question 2: How is the integration of a gamified metaphor using a social NPC and progression in software-based training in cognitive rehabilitation a) perceived in its implementation and b) does it affect emotional or motivational perceptions depending on corresponding player type?

In learning, gamification led to significant increases in cognitive, motivational and behavioral outcomes [58]. In motoric rehabilitation in home training, indications are found that addressing motivation may have a positively affect on motivation, adherence and training results [15]. As described above, a lot of training has a positive impact on effectiveness [67]. At the same time game elements are requested differently depending on the player type [63]. Therefore, it is important to examine how the use of elements affects training behavior, depending on whether the integrated elements fit the patient's type. This may have both, a positive but also a negative influence on the training duration. This leads us to Research Question 3: Does the implementation of the gamification elements NPC and progress influence the training duration in software-based training in cognitive rehabilitation depending on player type?

\subsection{Overview}

The research questions were investigated by expanding a clinical study [66] in patients with an acquired brain injury that underwent three weeks of computer-based training with or without additional game elements. In the presented work, we first consider the usercentred individual requests for game elements as a supplement to software-based training in cognitive rehabilitation (RQ1). For this, we consider the request for gamification elements depending on the patient's player type and compare the average requests of patients with the existing data of healthy persons. Second, we examine the subjectively perceived emotions and motivation (RQ2). For this, we consider as a basis the general perceived effect of the gamified training used and in detail on two player types. Third, we examine the behavior of the two player based on the performance in training duration (RQ3). Finally, we discuss the results and how they can be used in gamification frameworks and software-based cognitive training development. The results of this work contribute to the improvement of user-centred tailoring of gamification in software-based training in cognitive rehabilitation. 

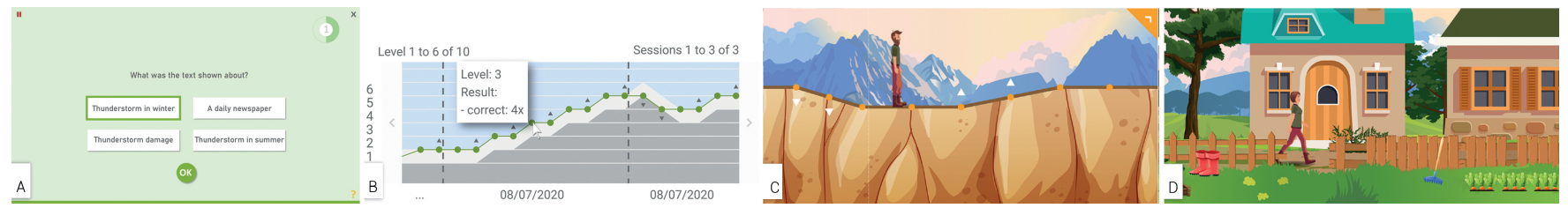

Figure 1: Training for verbal memory (A), line chart (B) and mountain ridge (C) for progress visualization and hiking path (D)

\section{METHODS}

\subsection{Clinical Study}

The data in this work was collected within a larger clinical study [66] targeting motivation in cognitive therapy after brain injury. Initial results indicate that gamification does not seem to influence behavior in general. However, there are indications of differences in motivational elements and the question arises about possible individual effects.

2.1.1 Study Design. $\mathrm{N}=83$ patients suffering from cognitive impairments after acquired brain injury gave written informed consent to participate in the study. They underwent outpatient cognitive rehabilitation and could conduct the study in terms of language, communication, cognitive and motor skills at home. They were in phase D to E based on the German rehabilitation system [53] and had mild to moderate cognitive impairments according to clinical and neuropsychological assessements (Montreal Cognitive Assessment [27]: $\mathrm{M}=25.98, \mathrm{SD}=2.61$; no significant differences between groups). Patients with severe deficits were not allowed to participate, as for them independent computer based training at home would not be reasonably possible. Participating patients were provided with access to a medically approved software-based training for three weeks. The number and duration of the training sessions were not fixed and could be chosen individually. However, it was recommended to train for 15 minutes at least twice a week, preferably more often. 64 patients completed the training phase successfully. The study included four phases: 1 . information, written consent and demographic data, 2. pre-survey identifying player types and requested game elements, 3. individual training phase, and 4. post-survey determining the effect of the gamification elements. The study was conducted multicentre and double-blind and was approved by the local's ethics committee (297/19-ek).

2.1.2 Basic and Gamified Cognitive training. We differentiate two groups within this work: Patients who used cognitive training without (group A) and cognitive training extended with game elements integrated in a metaphor (group B). Both groups conducted the same existing medically approved software training for verbal memory [18] as a web application (Fig. 1A). Within the training, texts were shown to the patient. The related information has to be memorized and reproduced in multiple-choice questions. Depending on the percentage of correct answers, the difficulty is adapted by adjusting the difficulty level from one to ten. The concept was wrapped around the training, i.e. before and after memory tasks' presentation. This ensured the therapeutic effectiveness of training. The requirement in the visualization of progress is realistic, not embellished, presentation to provide self-assessment. The information must not be presented in a too complicated way, not to overwhelm patients with cognitive impairments. As a basis and following current visualizations [19, 52], a line chart (Fig. 1B) of training performance was used. The green dots show the results of a specific task and the green line displays the overall performance and the progress of the training level. The grey area marks the levels below the current performance level, the white area marks the level in which the patient is classified, and the blue area marks the levels above. This visualization was presented to both groups. According to Hamari and Koivisto [21], the acceptance of gamification is influenced by both utilitarian and hedonic aspects. Based on the line visualization, the gamified metaphor of a hiking path was created in several brainstorming sessions by a multidisciplinary team of psychologists, computer scientists, and interaction designers focusing on gamification. Reasons for deciding on this metaphor included the transferability to the rehabilitation process and the accessibility for a wide range of patients. Nature tourism, especially hiking, offers opportunities for different age groups and abilities. The hiking path illustrates the patient's situation in a pictorial language in a meaningful way through a path towards a goal, mountain peak and successful rehabilitation. Within the therapy, the patient follows progress for which stamina, effort and small steps are necessary. As in rehabilitation, this involves setting a goal, but the process of achieving it is the largest part. A comparison between users or networking was not integrated because of data protection and the different rehabilitation potentials. The metaphor uses the core game elements of progress, integrated feedback, goal setting and a supporting NPC. Thus, core domains of progression and digital socialization are used. The gamified training was only used in group B. To realize the metaphor the line of the chart described above was transferred to a mountain ridge's corresponding setting (Fig. 1C). Both will be referred to in the following as the performance curve. To reinforce the feedback, patients can see in a second visualization how the landscape changes as they progress (Fig. 1D). This is independent of performance and shown after each training session. Along the way, milestones can be reached. Some are set by the system (e.g., completion of the 10th training session), but patients can also set personal goals. The display of ridge or path can be switched by using the orange arrow (Fig. 1C). The patient is accompanied by an NPC who walks along the path (Fig. 1D). The NPC does not represent the patient like an avatar, but is a person who accompanies the patient. The NPC is intended to help the patient to identify with the metaphor, support involvement in the training and to build up an emotional bond. With avatars, character identification has been shown to be related to the development of flow and openness to experience [60]. The design of the NPC was based on desired characteristics of a digital companion in rehabilitation, such as 'competent', 'motivating' or 'empathic' [16]. Patients can choose 
a woman or a man. The NPC interacts with the patient, e.g. by greeting and commenting on performance. In training, the patient can select equipment for the NPC from various objects for the hike to reach the mountain peak, symbolizing successful rehabilitation.

\subsection{Methods RQ1 - RQ3}

In this work, we exploratively consider the request and evaluation of game elements and effects in a comparison of different player types. The methods for RQ1 - RQ3 are described below. Due to dropouts of patients at different points in the study (e.g., due to technical and medical reasons), different numbers of patients are included in the analysis of the research questions. The respective number of patients included is stated in the results section for each research question. Exclusions are mainly based on missing data or anomalies / schematic answers or technical problems.

2.2.1 RQ 1: Player types and game elements. Player types and requested game elements were identified during the pre-survey. We used the Gamification User Types Hexad [3] and the corresponding existing questionnaire [65] to identify the patient's player types. It offers comparative data of healthy persons, a link to persuasive game strategies [50] and is particularly appropriate for gamification [20]. The questionnaire was translated into the language of the participants (german) by the authors. Responses were given on a 7-point Likert scale (1 (do not agree at all) - 7 (agree completely)) Participants can be assigned to several types based on equivalent results of the highest propensity. The player types identified permit the classification of patients as follows: primary type (highest score is in this type), non-primary type (highest score is not in this type and instead between second highest and lowest score) and least suitable type (lowest score is in this type). For distribution, we consider primary and least suitable type. For request of game elements, we used an adapted version of the questionnaire on Elements of Gameful Design (59 items, 5-point Likert scale (1 (I do not like it at all) - 5 (I like it very much)) [63]. In previous work, a cluster of eight categories (Socialization, Assistance, Immersion, Risk/Reward, Customization, Progression, Altruism and Incentive) showed the correlation between player types and categories [63]. The questionnaire was adapted to the context of rehabilitation because some items were difficult to understand for non-players. Three iterations assured the adaption quality with three middle-aged non-players and two cognitive rehabilitation experts to ensure comprehensibility and suitability. The changed items were compared to the original questionnaire to ensure that their meaning was preserved. One item (Social Competition) was transferred to the individual's performance (Self Competition). For the rating of the elements depending on the player type, we used the primary player type. For each element, the average ratings of all player types are stated. We then examined how many elements were rated exclusively positive, mixed (positive, neutral or negative) or exclusively negative by the player types. For the request in categories, we used the four top loading elements per category [63]. For 'Socialization', the fifth element (Social Status) was used due to the changed of 'Social Competition' to 'Self Competition'. We compared the average rating of the patients with existing data of healthy persons $[40,63]$.
2.2.2 RQ2 - Emotions and motivation. To determine the effect on the perception of individual player types of a gamified metaphor using a social NPC and progress visualization, ratings in the postsurvey were considered. All between-group comparisons were analyzed with the Mann-Whitney-U-test to detect significant differences (two-tailed, alpha $=0.05$ ). We collected the perception of the performance curves compared between group A and B in terms of comprehension, self-assessment and information content. The effect of gamification is context- and realization-dependent [20]. Therefore, we have considered one element describing emotional effect or the perception of the aspects relevant to the implementation of the metaphor. Collecting items or equipping an avatar or NPC are typical elements in games. In rehabilitation, however, the success of the patient is in the focus. Therefore, we considered whether supporting the NPC or receiving support was perceived as more positive by ratings in group B on a 5-point Likert scale ( $1=$ Not at all true, $5=$ Completely true). As a basis, we have considered the impact of the elements in general. In detail, we have analyzed two primary types: socialiser (because one core element of the gamified metaphor is based on social interaction with the NPC and socialization elements correlate most with socialisers) and achiever (as it correlates with progression in healthy persons most and is primarily motivated by competence perception [63]). We considered enjoyment and effort in both groups in the training phase based on a 5 -point Likert scale ( $1=$ none; $5=$ a lot). Based on elements of the Intrinsic Motivation Inventory (IMI; 7-point Likert scale: $1=$ not at all true; $7=$ very true) [28], we assessed the perceived competence.

2.2.3 RQ3 - Performance in training duration. To evaluate the effect of user-centered gamification on the behavioral level, the individual training duration was analyzed. For player types socialiser and achiever, we compare the training duration for both, within and between group A and B. For this, we considered the primary player types. The training duration is represented by median time spent with the cognitive training tasks. An unpaired t-test was used for significance analysis. The threshold for significance was set to 0.05 .

\section{RESULTS}

\subsection{RQ 1: Player types and game elements}

3.1.1 Results of patient data. 68 patients are completed the Player type questionnaire according to the Gamification User Types Hexad in the pre-survey $(\mathrm{f}($ female $)=29 ; \mathrm{m}($ male $)=38 ; \mathrm{d}$ (divers $)=1$, mean age $=47$ years, age range $=18-78$ years). Philanthropists are most $(27.59 \%)$, and disruptors are least represented in primary type (1.38\%). Regarding the least suitable type, the most are disruptors $(53.33 \%)$ and the least is free spirits $(1.11 \%)$. The distribution of all player types in patients is displayed in Fig. 2.

Data from 61 patients were analyzed regarding the adapted version of the questionnaire on Elements of Gameful Design ( $\mathrm{f}=28$, $\mathrm{m}=32, \mathrm{~d}=1$, mean age $=47$ years, age range $=18-78$ years). The detailed results can be found in the Fig. 5 in appendix A. The overall most requested element is 'Meaning/Progress' $(M=4.44, \mathrm{SD}=0.64)$ and the least 'Anchor juxtaposition' $(M=2.26, S D=0.96)$. For each element, the average ratings per player type are presented. 37 elements were rated higher than $M=3.0$ (neutral) by all types and are rather 


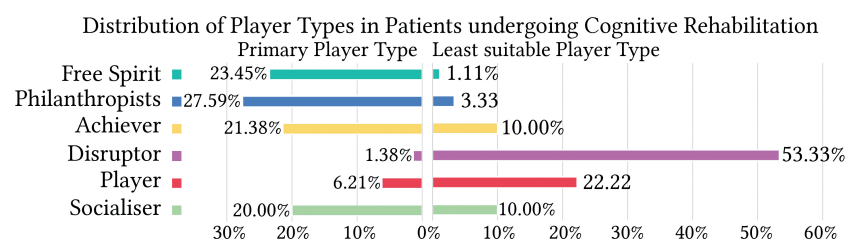

Figure 2: Distribution of Primary and Least Suitable Player Type in Patients

requested. 18 elements are neither clearly requested nor rejected. They received mixed ratings (positive, neutral, negative). 4 elements were rated lower than $\mathrm{M}=3.0$ by all types and are rather rejected. The ratings per category are shown in Table 1 . The assignment of the elements to the categories (based on [63]) is illustrated by icons in appendix A. The most requested category is 'Progression' $(\mathrm{M}=4.27, \mathrm{SD}=0.22)$ and the least is 'Socialization' $(\mathrm{M}=2.89, \mathrm{SD}=0.41)$

3.1.2 Analysis: Comparison of patient and healthy persons data. We compared RQ 1 results (distribution of player types and requested game elements) to existing data of healthy persons. The distribution of player types of patients was compared to existing data of healthy persons in a similar age group (40-49 years) according to Marczewski [40]. Deviations in the percentage distribution of primary player types are very low, at maximum about $5 \%$. Further, we compared the results of the requested game elements of patients with acquired cognitive impairment with existing data from healthy persons, according to Tondello et al. [63]. The main differences and similarities in categories and elements are shown in Fig. 3.

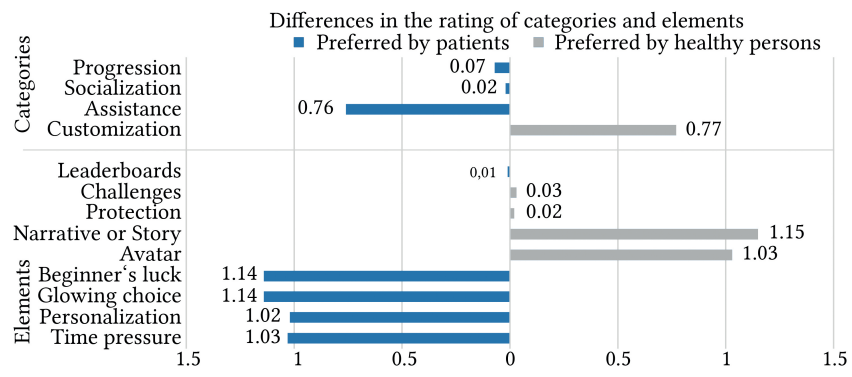

Figure 3: Main differences and similarities (mean values on a 5-point Likert Scale) of game elements and categories of healthy persons [63] and patients in cognitive rehabilitation

\subsection{RQ 2: Emotions and motivation}

53 patients filled out the pre- and post- survey questionnaires and completed the training phase and 43 are included in the analysis. 25 patients were in group $\mathrm{B}(\mathrm{f}=12, \mathrm{~m}=13$; mean age $=51$ years; age range $=18-78$ years, 12 primary socialisers, 12 primary achievers). 18 patients were in group $A(\mathrm{f}=7 ; \mathrm{m}=11$; mean age $=48$ years, age range $=23-63,8$ primary socialisers, 9 primary achievers). The rating of the performance curve's progress element (group A: line chart, group B: mountain ridge) is displayed in Table 2. Both were rated positively concerning training progress, comprehension, assess and details. No significant differences were found between the conditions. However, the mountain ridge was rated slightly lower. The perception of the gamified metaphor and the integrated elements (presented in group B) were rated positively. Detailed ratings are shown in Table 3.

Significant differences were found within the items between primary and non-primary type. Within socialisers a difference arose in the perception of supporting the NPC through clothes/equipment $(U=29, Z=-2.63805, p=0.0083)$. Primary socialisers perceived this as rather pleasant $(M=3.67, S D=0.75)$, non-primary socialisers rather not $(M=2.38, S D=1.00)$. In contrast, the difference of perception of getting support by the NPC was not significant $(U=54.5, Z=-1.25104$, $p=0.2113)$. Within achievers a difference arose in the perception of progression, displayed by the hike $(U=32.5, Z=-.2 .44768, p=0.01428)$. Primary achievers perceive this as more pleasant $(M=4.42, S D=0.86)$ than non-primary $(M=3.38, S D=1.08)$. No other group differences were detected. Regarding emotions and motivation the overall perceived enjoyment was significantly higher in the gamified version of the cognitive training. Perceived effort and competence were not significant different. In detail, however primary socialisers perceived significantly more enjoyment and primary achievers significantly more competence in gamified than in the non-gamified training. The $p$-values of the significance analysis overall and for individual player types are presented in Table 4.

\subsection{RQ 3: Performance in training duration}

59 patients filled out the player type questionnaires, completed the training phase and were included in the analysis. 29 patients were in group $\mathrm{B}(\mathrm{f}=10 ; \mathrm{m}=19 ;$ mean age $=49$ years, age range $=18-78$ years, 12 primary socialisers, 14 are primary achievers). 30 patients were in group $A(f=14, m=16$; mean age $=48$ years, age range $=19-70$ years, 13 primary socialisers, 13 primary achievers). Comparing the overall training duration revealed no significant differences between group A and B (group A: md=207 min; group B: $\mathrm{md}=194$ min.; $t=-0.42827, p=0.670069$ ). For an examination of player typedependent behavior, socialisers and achievers were considered. The median training duration of both types in groups A and B is shown in Fig. 4. Socialisers with the gamified metaphor and NPC trained the most ( $\mathrm{md}=267.5 \mathrm{~min}, \mathrm{R}=792$ ). There was a significant difference in training duration in group B between primary and non-primary socialisers $(t=-2.19206 ; p=0.037176)$. No significant differences were found between primary and non-primary achievers in group B $(t=-1.26938, p=0.21514)$ or within the types.

\section{DISCUSSION}

\subsection{RQ 1: Player types and game elements}

Our results indicate that patients and healthy persons show similar distributions of player types and cognitive impairment does not lead to substantial changes in motivational processes after brain injury. However, 'Assistance' is requested more by patients than by healthy persons. Although subjects of a comparable age range were considered, the healthy persons were younger on average (27 years) than the patients ( 47 years). Usually the proportion of philantropists increases with increasing age [40]. However, if the difference found were only due to age, categories that correlate with philantropists (such as Immersion, Progression or Altruism [63]) should be more 
Table 1: Requested game elements of patients by categories. Highest ratings are printed in bold, lowest in italic. Philanthropist (Ph), Free Spirit (FS), Achiever (A), Socialiser (S), Player (Pl), Disruptor (D). Values are stated in mean (standard deviation).

\begin{tabular}{lccccccc}
\hline Name & Overall (SD) & $\mathrm{Ph}(\mathrm{SD})$ & $\mathrm{FS}(\mathrm{SD})$ & $\mathrm{A}(\mathrm{SD})$ & $\mathrm{S}(\mathrm{SD})$ & $\mathrm{Pl}(\mathrm{SD})$ & $\mathrm{D}(\mathrm{SD})$ \\
\hline Progression & $\mathbf{4 . 2 7}(\mathbf{0 . 2 2})$ & $\mathbf{4 . 2 0 ( \mathbf { 0 . 2 5 } )}$ & $\mathbf{3 . 9 4 ( \mathbf { 0 . 1 9 } )}$ & $\mathbf{4 . 0 5 ( \mathbf { 0 . 3 0 } )}$ & $\mathbf{4 . 1 8 ( \mathbf { 0 . 0 8 } )}$ & $\mathbf{4 . 3 6 ( \mathbf { 0 . 2 3 } )}$ & $\mathbf{4 . 7 5 ( \mathbf { 0 . 4 3 } )}$ \\
Assistance & $3.67(0.82)$ & $3.67(0.83)$ & $3.54(0.70)$ & $3.49(0.81)$ & $3.68(0.76)$ & $3.69(0.81)$ & $3.50(0.87)$ \\
Risk/Reward & $3.61(0.44)$ & $3.60(0.56)$ & $3.51(0.62)$ & $3.68(0.52)$ & $3.45(0.51)$ & $3.53(0.40)$ & $3.63(1.24)$ \\
Immersion & $3.62(0.18)$ & $3.54(0.22)$ & $3.38(0.31)$ & $3.60(0.25)$ & $3.56(0.27)$ & $3.78(0.32)$ & $3.75(0.43)$ \\
Incentive & $3.59(0.06)$ & $3.54(0.12)$ & $3.42(0.07)$ & $3.73(0.15)$ & $3.35(0.08)$ & $3.83(0.23)$ & $3.50(0.35)$ \\
Altruism & $3.36(0.05)$ & $3.34(0.07)$ & $3.23(0.07)$ & $3.51(0.20)$ & $3.40(0.12)$ & $3.39(0.24)$ & $3.75(0.25)$ \\
Customization & $3.22(0.34)$ & $3.19(0.41)$ & $3.07(0.31)$ & $3.29(0.35)$ & $3.09(0.28)$ & $3.44(0.45)$ & $2.75(0.25)$ \\
Socialization & $2.89(0.41)$ & $2.81(0.42)$ & $2.69(0.32)$ & $3.06(0.46)$ & $2.92(0.37)$ & $3.22(0.47)$ & $3.38(0.41)$ \\
\hline
\end{tabular}

Table 2: Rating and p-values of the Performance Curve as a Line Chart (Group A) and additional Mountain Ridge (Group B). Significant values $(\mathrm{p}<.05)$ are printed in bold. Items were rated on a 5-point Likert scale ( $1=$ Not at all true, $5=$ Completely true). Values (A and B) are stated in mean (standard deviation), p-value is based on the difference between group $A$ and $B$.

\begin{tabular}{lccc}
\hline Item & $\begin{array}{c}\text { Overall } \\
\text { Group A }\end{array}$ & $\begin{array}{c}\text { Overall } \\
\text { Group B }\end{array}$ & $\begin{array}{c}\text { Group difference } \\
\text { p-value }\end{array}$ \\
\hline $\begin{array}{l}\text { Training progress: I have paid a lot of attention to the visualization } \\
\text { of my current training progress in the performance curve. }\end{array}$ & $4.00(0.88)$ & $3.72(1.00)$ & 0.42372 \\
$\begin{array}{l}\text { Comprehension: It was easy for me to comprehend the information } \\
\text { shown in the performance curve. }\end{array}$ & $4.00(1.03)$ & $3.88(0.82)$ & 0.4593 \\
$\begin{array}{l}\text { Assess: Information shown in the performance curve helped me to } \\
\text { better assess my own performance. }\end{array}$ & $3.94(0.97)$ & $3.72(0.83)$ & 0.37346 \\
$\begin{array}{l}\text { Details: I would like to receive detailed information about my } \\
\text { current training to be able to better assess my current performance. }\end{array}$ & $4.11(0.81)$ & $4.04(0.77)$ & 0.7414 \\
\hline
\end{tabular}

Table 3: Rating of the elements of the gamified metaphor in group B. Items were rated on a 5-point Likert scale (1=Not at all true, $5=$ Completely true). Values are stated in mean (standard deviation).

\begin{tabular}{lccc}
\hline Item & Overall & $\begin{array}{c}\text { Primary } \\
\text { Socialiser }\end{array}$ & $\begin{array}{c}\text { Primary } \\
\text { Achiever }\end{array}$ \\
\hline Metaphor: I like the idea of seeing my rehabilitation as a hike. & $4.16(0.78)$ & $4.17(0.80)$ & $4.33(0.75)$ \\
Goal: It felt good to formulate a goal and work towards it. & $4.32(0.68)$ & $4.33(0.75)$ & $4.50(0.65)$ \\
Visualization: I liked that the performance curve was shown & & & \\
as a mountain ridge. & $4.08(0.80)$ & $4.08(0.86)$ & $4.33(0.75)$ \\
Progress: The trail was a good way to see what I have already achieved. & $3.88(1.11)$ & $4.25(0.60)$ & $4.42(0.86)$ \\
Milestones: I was very happy about reaching milestones. & $4.36(0.69)$ & $4.42(0.76)$ & $4.50(0.65)$ \\
NPC: It was pleasant to have the person by my side. & $3.56(0.75)$ & $3.67(0.62)$ & $3.75(0.83)$ \\
NPC: It was great to support the person through the clothes / equipment & & & \\
we got in training. & $3.00(1.10)$ & $3.67(0.75)$ & $3.34(1.03)$ \\
NPC: It was great to get support from the person. & $3.52(0.90)$ & $3.83(0.80)$ & $3.92(0.76)$ \\
\hline
\end{tabular}

Table 4: Perceived emotions. Significant values $(\mathbf{p}<.05)$ are printed in bold. Values are stated in mean (standard deviation), p-value is based on the difference between group A and B for: overall, primary socialisers and primary achievers.

\begin{tabular}{l|ccc|cccccc}
\hline & \multicolumn{3}{|c}{ Overall } & \multicolumn{3}{c}{ Primary Socialiser } & \multicolumn{3}{c}{ Primary Achiever } \\
Item & Group A & Group B & p-value & Group A & Group B & p-value & Group A & Group B & p-value \\
\hline Enjoyment & $3.61(0.95)$ & $4.28(0.72)$ & $\mathbf{0 . 0 3 1 5 6}$ & $3.63(0.70)$ & $4.50(0.65)$ & $\mathbf{0 . 0 2 5 1}$ & $3.56(1.17)$ & $4.33(0.85)$ & 0.13622 \\
Effort & $3.56(0.60)$ & $3.36(0.89)$ & 0.71138 & $3.38(0.48)$ & $3.42(0.64)$ & 0.8181 & $3.67(0.67)$ & $3.58(0.64)$ & 0.9681 \\
Competence & $4.61(1.16)$ & $5.00(0.87)$ & 0.33204 & $5.00(0.75)$ & $5.04(0.88)$ & 0.8181 & $4.28(0.89)$ & $5.17(0.85)$ & $\mathbf{0 . 0 3 9 4}$ \\
\hline
\end{tabular}



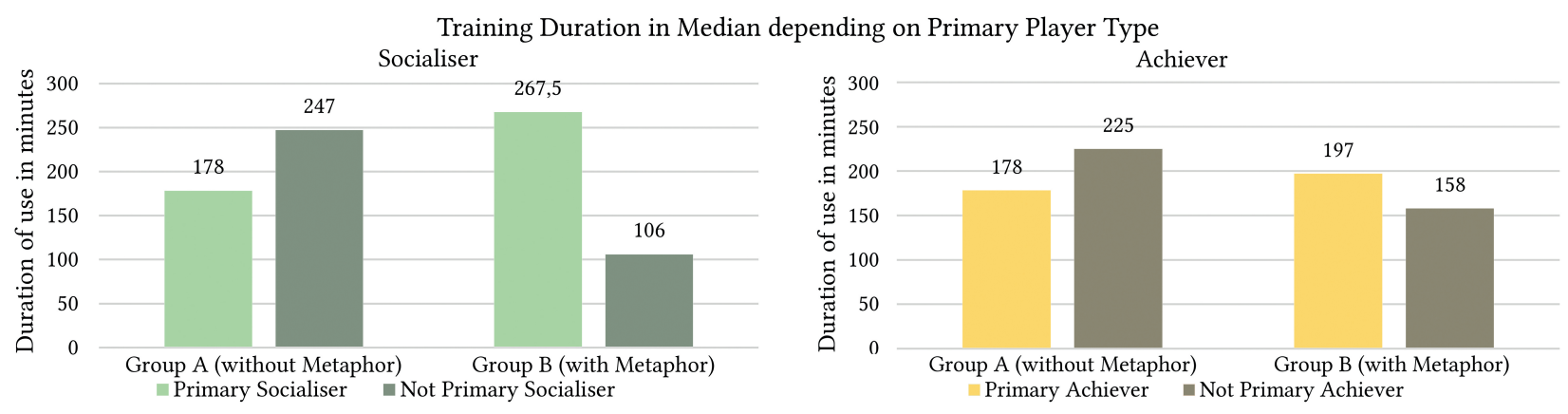

Figure 4: Training Duration depending in Primary Player Type Socialiser and Achiever

requested by patients. This is not the case. In contrast, the element 'Narrative or Story', which is assigned to immersion, is requested less by patients than by healthy persons. This reinforces the contextual relevance when using gamification [20, 22]. However, other factors such as the way of using digital media may also influence the requests. Designing new cognitive intervention programs is time consuming and cost-intensive. Therefore, gamification elements are usually additionally integrated into existing software [43]. In line with previous research [45, 50], our results support the implementation phase of gamification in software-based cognitive training early in the development. The 37 elements, requested by all player types can generally be used as a basis. In a second step, these and additional 18 mixed-rated elements may be implemented for individual player types. Through step-by-step integration, it is possible to focus on an iterative implementation and optimization of the elements [45]. However, for use in practice, it should be kept in mind that the use of several similar strategies probably won't reinforce behavioral change [30]. Also, elements may be rejected even though they correspond to the player type. The option to turn them off can motivate instead [37]. This shows that the evaluation of individual gamification elements should not be considered entirely based on overall average of mixed participants. Ratings of different types, individual evaluations or use-specific evaluations may deviate.

\subsection{RQ 2: Emotions and motivation}

Both the visualization of the line chart and the transfer to a mountain ridge were rated rather positively. No significant differences were found, indicating that the integration of the metaphor may not affect the perception of feedback. However, the mountain ridge was rated slightly lower. This should be considered further in detail and a larger sample size. Thereby it also should be analyzed whether and if so which aspects (such as type of visualization or diagrams or complexity) might influence. The gamified metaphor was rated positively overall and led to more enjoyment. In contrast, the perceived effort was similar in the gamified and non-gamified versions, indicating that gamification elements neither reduce nor increase exertion in task execution. A closer look at the player types when using the gamified training revealed that perceived changes in emotions (i.e., enjoyment) and motivation (i.e., competence) were limited to the respective player type and did not influence the patients in general (i.e., despite improved competence achievers did not feel more enjoyment). These findings replicate the observations in healthy persons for learning [58], games for change [47] and other rehabilitation domains [8], showing that perceived emotion and motivation can be influenced by gamification. The different effects within the player types highlight the relevance of tailoring game elements to the characteristics of the users. Our study's gamified metaphor was designed as a system with elements connected to each other to create a holistic valuable user experience [21]. Jagušt et al. suggest that the effect of gamification also depends on the combination of elements [25]. For user-centred tailoring of game elements in software-based cognitive training, the possibility to activate or deactivate specific game elements depending on individual needs is highly recommended [43]. This shows that the use of gamification elements may be planned specifically to address different emotions in different types.

\subsection{RQ 3: Performance in training duration}

Despite the increased emotions (RQ2) no significant effect was found regarding the performance in training duration in the gamified vs non-gamified version. There were no differences neither in the overall comparison, which confirms the initial results [66], nor in the individual consideration of the player types. Here the question arises whether the content-related stimulus of the training and the integrated game elements were not intense enough or the ultimate goal of the intervention - improvement of cognitive functions - may be so crucial that the patients executed the tasks intrinsically motivated even without reinforcement through game elements [36]. Also in other gamified rehabilitation training, no effects on efficacy were found [8]. However, we found evidence that game elements, which match the individual player type, are able to actually increase perseverance and training performance within gamified training. Social elements are rather low requested (RQ1). These primarily refer to interactions with real persons and the digital NPC implemented in our study seems to have left a different impression, resulting in a relevant impact on motivation [20]. Within training with the gamified metaphor, patients trained significantly longer, when they were primarily socialisers. As training duration represents one of the main reasons for efficacy [67], we showed that the tailoring of game elements in cognitive rehabilitation is highly relevant. In terms of desired behavioral changes, the emergence of such effects should be considered more closely to achieve positive and avoid negative effects [29,48]. This should not 
only be considered between gamified and non-gamified, but also within gamified systems. However, achievers who usually request progression did not differ significantly regarding their overall training duration. We suggest the following explanations for the missing impact on the behavioral measure: First, the gamified metaphor included two elements requested differently by all patients before the training. While socialization was classified as least appealing, progression was the most wanted. That implies that user-centred tailoring is particularly relevant for low-requested or polarizing elements. Second, research on persuasive strategies showed that for socialisers significant relationships between player type and among others goalsetting, cooperation and self-monitoring / feedback were found [50], but not for achievers. The results support that within gamified applications, elements suitable for the type should be used to support desired behavior.

\subsection{General Discussion}

Gamification in health is intended to improve perceived emotions and motivation on the one hand and the targeted behavioral change on the other. The highest request for 'Progression' and 'Assistance' is in line with patients' desire to get support and improve their cognitive abilities. The possibility of impaired social cognitive functioning due to acquired brain injury [33] may have had a concomitant effect on the rejection of 'socialization' elements. However, positive emotions and motivation have been shown in socialisers. Gamified training using elements of progress visualization and socialization, showed relevant impact on emotions, motivation, and behavior for patients in software-based training during cognitive rehabilitation. Notably, the effects were mostly dependent on the individual player type, indicating that user-centred tailoring is particularly important in this user group. This has been shown in the different emotional effect in enjoyment and competence on socialisers and achievers and in detail by the example that primary socialisers tend to perceive it as rather pleasant to support the NPC, whereas non primary socialisers find it rather unpleasant. However, in rehabilitation, the focus is on receiving support. Thus, tailored elements may potentially address additional user needs that are not the focus of therapy, but are individually relevant for the patient's motivation. Goals of gamification, perceived emotions and motivation, and behavior are relevant for training efficacy [12]. This corroborates the approach to focus on the patients' needs in the conception of gamification frameworks [45]. Hallifax et al. [61] point out that when planning gamification, the dominant player type may not be sufficient to categorise the users' preferences. User's initial motivation profile may also used for dual adaptation to optimize the tailoring of the elements. Due to the observed differences in training duration depending on requested and declined game elements, it may be relevant to consider least represented types or elements that are low requested but fit to the type. In cognitive rehabilitation, additional factors such awareness of the disease, general compliance or goals could be considered. For player type-related effects other player type models that consider goal setting may be included [62]. For the acceptance of gamification in the context of rehabilitation, further aspects, such as visual style, onboarding, or depth of serious or gameful to playful elements, or complexity should be considered. The results support the relevance to define in the conception of gamification whether the requests of the users, emotions and motivation or the effectiveness shall be addressed.

\section{LIMITATIONS AND FUTURE WORK}

In this study, patients from Germany in outpatient therapy with mild to moderate cognitive impairment were recruited. The results provide orientation, but generalization or transferability to other countries, rehabilitation systems, or application fields cannot be ensured. The questionnaires used were adapted to the patients' language and the rehabilitation context. This should be considered when interpreting the comparison with data of healthy persons. Due to the small number of the player type 'Disruptor' among the patients corresponding results can only considered to a limited extent. The questionnaires (RQ1) demonstrate only the request of game elements, not the actual effect on motivation during training. Also, by answering the questionnaire on gamification elements, there is the possibility of influencing the expectations regarding the training. The sample of the study was rather small within the subdivision into the individual groups and in RQ 2 and 3 only two player types (socialiser and achiever) were considered. A different study design or a different way of implementing a metaphor may affect the results. Further research is needed to investigate the differential impact of player types on performance in patients. Due to reporting averaged data, individual preferences may deviate from these results. Due to the combination of the elements' progression and NPC, separating their distinctive effects is not possible. Likewise, some overlap between various player types is given because patients can tend to several types [62], even if they have partly different intensity levels. In line with Hallifax et al. [20] future work should consider reciprocal effects between elements and / or tendencies for different types of players. As this study was conducted in the context of a larger clinical study [66], some patients received additional advice for strategies during task completion and slight corrections were implemented. An additional effect of strategy teaching may exist but does not impact results due to equal distribution of them in group A and B. Our study represents the requests and behavior of patients. When selecting game elements, the planned training content's therapeutic suitability should also be considered. Future applications could focus on emotional support and more specific tailoring of gamified elements with dual tailoring.

\section{CONCLUSION}

In this paper, we investigated player type dependent 1) the request for game elements of patients in software-based training during home-based cognitive rehabilitation, 2) perceived emotional and motivational aspects in non-gamified and gamified training, and 3) the behavioral effect on performance (i.e., training duration). All player types of patients in cognitive rehabilitation request almost two-thirds of the elements, and others are considered differently or rejected. Thus, we recommend implementing items identified as generally pleasant first. Later, tailored gamification of additional game elements may be integrated step by step. The gamified training led to more enjoyment overall. In detail, however, the player types reacted differently: Primary achievers perceived a higher level of competence, primary socialisers perceived more enjoyment during training. Despite perceived emotions and motivation, there 
was only a limited effect on behavioral performance of player types. Although no differences were found in training duration between gamified and non-gamified training, in gamified training, primary socialisers trained significantly longer than non-primary socialisers. This indicates a possible dependence of player type, suitability of the elements used and training duration, emphasising the importance of user-centred tailoring of gamification. Overall, the results indicate that gamification is suitable in cognitive rehabilitation and may lead to emotional, motivational and behavioral improvements. Here it is relevant to specify in the conception which aspect is to be addressed. It is also relevant to select appropriate elements depending on the individual player type to maximise the benefits and avoid adverse effects. The results of the present study contribute to improving the selection and use of game elements in a user-centered and tailored way. This may support the optimization of software-based training in cognitive rehabilitation.

\section{ACKNOWLEDGMENTS}

This work was funded by the European Regional Development Fund under the operation number ZS/2016/04/78123 and number ZS/2017/01/83843 as part of the initiative 'Sachsen-Anhalt WISSENSCHAFT Schwerpunkte'. It was also funded by the European Social Fund (ESF) of Saxony-Anhalt (under the operation number CCI 2014DE05SFOP013) as part of the initiative 'Sachsen-Anhalt WISSENSCHAFT Chancengleichheit' with the program title 'FEMPower'.

\section{REFERENCES}

[1] Gazihan Alankus, Rachel Proffitt, Caitlin Kelleher, and Jack Engsberg. 2011. Stroke therapy through motion-based games: a case study. ACM Transactions on Accessible Computing (TACCESS) 4, 1 (2011), 1-35.

[2] Ahmed Allam, Zlatina Kostova, Kent Nakamoto, and Peter Johannes Schulz. 2015 The effect of social support features and gamification on a Web-based intervention for rheumatoid arthritis patients: randomized controlled trial. Fournal of medical Internet research 17, 1 (2015), e14.

[3] M Andrzej. 2015. Even Ninja Monkeys Like to Play: Gamification, Game Thinking and Motivational Design.

[4] Richard Bartle. 1996. Hearts, clubs, diamonds, spades: Players who suit MUDs. Journal of MUD research 1, 1 (1996), 19.

[5] Gerard Beenen, Kimberly Ling, Xiaoqing Wang, Klarissa Chang, Dan Frankowski, Paul Resnick, and Robert E Kraut. 2004. Using social psychology to motivate contributions to online communities. In Proceedings of the 2004 ACM conference on Computer supported cooperative work. 212-221.

[6] Paula Bräuer and Athanasios Mazarakis. 2019. Badges or a leaderboard? How to gamify an augmented reality warehouse setting.. In Proceedings of the $3 \mathrm{rd}$ International GamiFIN Conference. 229-240.

[7] James William Burke, MDJ McNeill, Darryl K Charles, Philip J Morrow, Jacqui H Crosbie, and Suzanne M McDonough. 2009. Optimising engagement for stroke rehabilitation using serious games. The Visual Computer 25, 12 (2009), 1085.

[8] Martí de Castro-Cros, Marc Sebastian-Romagosa, Javier Rodríguez-Serrano, Eloy Opisso, Manel Ochoa, Rupert Ortner, Christoph Guger, and Dani Tost. 2020. Effects of gamification in BCI functional rehabilitation. Frontiers in neuroscience $14(2020), 882$.

[9] Teresa de la Hera Conde-Pumpido. 2017. Persuasive gaming: Identifying the different types of persuasion through games. International fournal of Serious Games 4, 1 (2017), 31-39.

[10] Aijse W De Vries, Jaap H Van Dieën, Vero Van Den Abeele, and Sabine MP Verschueren. 2018. Understanding motivations and player experiences of older adults in virtual reality training. Games for health journal 7, 6 (2018), 369-376.

[11] Sebastian Deterding, Rilla Khaled, Lennart E Nacke, and Dan Dixon. 2011. Gamification: Toward a definition. In CHI 2011 gamification workshop proceedings, Vol. 12. Vancouver BC, Canada.

[12] Sandra Dörrenbächer, Philipp M Müller, Johannes Tröger, and Jutta Kray. 2014 Dissociable effects of game elements on motivation and cognition in a taskswitching training in middle childhood. Frontiers in psychology 5 (2014), 1275.

[13] Miriam Erez. 1977. Feedback: A necessary condition for the goal settingperformance relationship. Journal of Applied psychology 62, 5 (1977), 624.
[14] Gunther Eysenbach. 2005. The law of attrition. Fournal of medical Internet research 7,1 (2005), e11.

[15] Gerard G Fluet, Qinyin Qiu, Jigna Patel, Amanda Cronce, Alma S Merians, and Sergei V Adamovich. 2019. Autonomous use of the home virtual rehabilitation system: A feasibility and pilot study. Games for healt journal 8, 6 (2019), 432-438.

[16] Mareike Gabele, Andrea Thoms, Julian Alpers, Steffi Hußlein, and Christian Hansen. 2019. Non-player character as a companion in cognitive rehabilitation for adults - Characteristics and representation. In Proceedings of the 3rd International GamiFIN Conference. 130-141.

[17] Thomas A Glass, David B Matchar, Michael Belyea, and JR Feussner. 1993. Impact of social support on outcome in first stroke. Stroke 24, 1 (1993), 64-70.

[18] Hasomed GmbH. 2018. VerbalMemory. Retrieved July 7, 2020 from https: //hasomed.de/wp-content/uploads/hasomed-fileadmin/RehaCom/Mediathek/ Handbuecher/Englisch/RehaCom_UserManual_Verbal_Memory.pdf

[19] Hasomed GmbH. 2020. RehaCom - Cognitive Therapy. Retrieved January 22, 2021 from https://hasomed.de/en/products/rehacom/\#3n9stm6n2Collapse0

[20] Stuart Hallifax, Audrey Serna, Jean-Charles Marty, Guillaume Lavoué, and Elise Lavoué. 2019. Factors to Consider for Tailored Gamification. In Proceedings of the Annual Symposium on Computer-Human Interaction in Play. 559-572.

[21] Juho Hamari and Jonna Koivisto. 2015. Why do people use gamification services? International fournal of Information Management 35, 4 (2015), 419-431.

[22] Juho Hamari, Jonna Koivisto, and Harri Sarsa. 2014. Does Gamification Work? - A Literature Review of Empirical Studies on Gamification. 2014 47th Hawaii International Conference on System Sciences (2014), 3025-3034.

[23] Ya-Xuan Hung, Pei-Chen Huang, Kuan-Ta Chen, and Woei-Chyn Chu. 2016. What do stroke patients look for in game-based rehabilitation: a survey study. Medicine 95, 11 (2016).

[24] Kai Huotari and Juho Hamari. 2017. A definition for gamification: anchoring gamification in the service marketing literature. Electronic Markets 27, 1 (2017), 21-31.

[25] Tomislav Jagušt, Ivica Botički, and Hyo-Jeong So. 2018. Examining competitive, collaborative and adaptive gamification in young learners' math learning. Computers \& education 125 (2018), 444-457.

[26] Daniel Johnson, Sebastian Deterding, Kerri-Ann Kuhn, Aleksandra Staneva, Stoyan Stoyanov, and Leanne Hides. 2016. Gamification for health and wellbeing: A systematic review of the literature. Internet interventions 6 (2016), 89-106.

[27] Parunyou Julayanont, Sookjaroen Tangwongchai, Solaphat Hemrungrojn, Chawit Tunvirachaisakul, Kammant Phanthumchinda, Juntanee Hongsawat, Panida Suwichanarakul, Saowaluck Thanasirorat, and Ziad S Nasreddine. 2015. The montreal cognitive assessment-basic: A screening tool for mild cognitive impairment in illiterate and low-educated elderly adults. Fournal of the American Geriatrics Society 63, 12 (2015), 2550-2554.

[28] Frederick H Kanfer. 1986. Implications of a self-regulation model of therapy for treatment of addictive behaviors. In Treating addictive behaviors. Springer, $29-47$.

[29] Maurits Kaptein, Boris De Ruyter, Panos Markopoulos, and Emile Aarts. 2012. Adaptive persuasive systems: a study of tailored persuasive text messages to reduce snacking. ACM Transactions on Interactive Intelligent Systems (TiiS) 2, 2 (2012), 1-25.

[30] Maurits Kaptein, Steven Duplinsky, and Panos Markopoulos. 2011. Means based adaptive persuasive systems. In Proceedings of the SIGCHI conference on human factors in computing systems. 335-344.

[31] Maurits Kaptein, Panos Markopoulos, Boris de Ruyter, and Emile Aarts. 2009. Can you be persuaded? individual differences in susceptibility to persuasion. In IFIP conference on human-computer interaction. Springer, 115-118.

[32] Elaine Kearney, Sanjana Shellikeri, Rosemary Martino, and Yana Yunusova. 2019. Augmented visual feedback-aided interventions for motor rehabilitation in Parkinson's disease: a systematic review. Disability and rehabilitation 41, 9 (2019), 995-1011.

[33] Michelle Kelly, Skye McDonald, and Matthew HJ Frith. 2017. Assessment and rehabilitation of social cognition impairment after brain injury: surveying practices of clinicians. Brain Impairment 18, 1 (2017), 11-35.

[34] Rilla Khaled, Pippin Barr, James Noble, Ronald Fischer, and Robert Biddle. 2007. Fine tuning the persuasion in persuasive games. In International conference on persuasive technology. Springer, 36-47.

[35] Jonna Koivisto and Juho Hamari. 2019. The rise of motivational information systems: A review of gamification research. International fournal of Information Management 45 (2019), 191-210.

[36] Andrea Kusec, Diana Velikonja, Carol DeMatteo, and Jocelyn E Harris. 2019. Motivation in rehabilitation and acquired brain injury: can theory help us understand it? Disability and rehabilitation 41, 19 (2019), 2343-2349.

[37] Pascal Lessel, Maximilian Altmeyer, Lea Verena Schmeer, and Antonio Krüger. 2019. "Enable or Disable Gamification?" Analyzing the Impact of Choice in a Gamified Image Tagging Task. In Proceedings of the 2019 CHI Conference on Human Factors in Computing Systems. 1-12.

[38] James J Lin, Lena Mamykina, Silvia Lindtner, Gregory Delajoux, and Henry B Strub. 2006. Fish'n'Steps: Encouraging physical activity with an interactive computer game. In International conference on ubiquitous computing. Springer, 
261-278.

[39] Edwin A Locke and Gary P Latham. 1990. A theory of goal setting \& task performance. Prentice-Hall, Inc.

[40] Andrzej Marczewski. 2018. Considering Age and other factors in Gamification. Retrieved January 22, 2021 from https://www.gamified.uk/2018/06/20/consideringage-and-other-factors-in-gamification

[41] Athanasios Mazarakis and Paula Bräuer. 2017. Welche Gamification motiviert? Ein Experiment zu Abzeichen, Feedback, Fortschrittsanzeige und Story. GeNeMe'17-Gemeinschaften in Neuen Medien, 20. Tagung (2017), 246-255.

[42] Shanthi Mendis. 2013. Stroke disability and rehabilitation of stroke: World Health Organization perspective. International fournal of stroke 8, 1 (2013), 3-4.

[43] Baptiste Monterrat, Michel Desmarais, Elise Lavoué, and Sébastien George. 2015 A player model for adaptive gamification in learning environments. In International conference on artificial intelligence in education. Springer, 297-306.

[44] Alberto Mora, Daniel Riera, Carina Gonzalez, and Joan Arnedo-Moreno. 2015. A literature review of gamification design frameworks. In 2015 7th International Conference on Games and Virtual Worlds for Serious Applications (VS-Games). IEEE $1-8$.

[45] Benedikt Morschheuser, Lobna Hassan, Karl Werder, and Juho Hamari. 2018. How to design gamification? A method for engineering gamified software. Information and Software Technology 95 (2018), 219-237.

[46] Lennart E Nacke, Chris Bateman, and Regan L Mandryk. 2014. BrainHex: A neurobiological gamer typology survey. Entertainment computing 5, 1 (2014), $55-62$.

[47] Rita Orji, Regan L Mandryk, and Julita Vassileva. 2017. Improving the efficacy of games for change using personalization models. ACM Transactions on ComputerHuman Interaction (TOCHI) 24, 5 (2017), 1-22.

[48] Rita Orji, Regan L Mandryk, Julita Vassileva, and Kathrin M Gerling. 2013. Tailoring persuasive health games to gamer type. In Proceedings of the sigchi conference on human factors in computing systems. 2467-2476.

[49] Rita Orji and Karyn Moffatt. 2018. Persuasive technology for health and wellness: State-of-the-art and emerging trends. Health informatics journal 24, 1 (2018), 66-91.

[50] Rita Orji, Gustavo F Tondello, and Lennart E Nacke. 2018. Personalizing persuasive strategies in gameful systems to gamification user types. In Proceedings of the 2018 CHI Conference on Human Factors in Computing Systems. 1-14.

[51] Amalia Ortiz, María del Puy Carretero, David Oyarzun, Jose Javier Yanguas, Cristina Buiza, M Feli Gonzalez, and Igone Etxeberria. 2007. Elderly users in ambient intelligence: Does an avatar improve the interaction? In Universal access in ambient intelligence environments. Springer, 99-114.

[52] Inc. Pearson Education. 2017. Cogmed. Retrieved January 22, 2021 from https://play.google.com/store/apps/details?id=air.com.pearsoned.cogmed $\mathrm{rm} \& \mathrm{hl}=\mathrm{de} \& \mathrm{gl}=\mathrm{US}$

[53] Verband Deutscher Rentenversicherungsträger. 1995. Phaseneinteilung in der neurologischen Rehabilitation. Rehabilitation 34 (1995), 119-127.

[54] Karen Robson, Kirk Plangger, Jan H Kietzmann, Ian McCarthy, and Leyland Pitt. 2015. Is it all a game? Understanding the principles of gamification. Business horizons 58, 4 (2015), 411-420.

[55] William Ruberman, Eve Weinblatt, Judith D Goldberg, and Banvir S Chaudhary. 1984. Psychosocial influences on mortality after myocardial infarction. New England fournal of Medicine 311, 9 (1984), 552-559.

[56] Richard M Ryan and Edward L Deci. 2000. Self-determination theory and the facilitation of intrinsic motivation, social development, and well-being. American psychologist 55, 1 (2000), 68.

[57] Michael Sailer, Jan Ulrich Hense, Sarah Katharina Mayr, and Heinz Mandl. 2017. How gamification motivates: An experimental study of the effects of specific game design elements on psychological need satisfaction. Computers in Human Behavior 69 (2017), 371-380.

[58] Michael Sailer and Lisa Homner. 2020. The gamification of learning: A metaanalysis. Educational Psychology Review.

[59] Lamyae Sardi, Ali Idri, and José Luis Fernández-Alemán. 2017. A systematic review of gamification in e-Health. Journal of biomedical informatics 71 (2017), 31-48.

[60] Alistair Raymond Bryce Soutter and Michael Hitchens. 2016. The relationship between character identification and flow state within video games. Computers in Human Behavior 55 (1 Feb. 2016), 1030-1038. https://doi.org/10.1016/j.chb. 2015.11.012

[61] Hallifax Stuart, Elise Lavoué, and Audrey Serna. 2020. To tailor or not to tailor gamification? An analysis of the impact of tailored game elements on learners' behaviours and motivation. In 21th International Conference on Artificial Intelligence in Education.

[62] Gustavo F Tondello, Karina Arrambide, Giovanni Ribeiro, Andrew Jian-lan Cen, and Lennart E Nacke. 2019. "I don't fit into a single type": A Trait Model and Scale of Game Playing Preferences. In IFIP Conference on Human-Computer Interaction. Springer, 375-395.

[63] Gustavo F Tondello, Alberto Mora, and Lennart E Nacke. 2017. Elements of gameful design emerging from user preferences. In Proceedings of the Annual Symposium on Computer-Human Interaction in Play. 129-142.
[64] Gustavo F Tondello and Lennart E Nacke. 2020. Validation of User Preferences and Effects of Personalized Gamification on Task Performance. Frontiers in Computer Science 2 (2020), 29.

[65] Gustavo F Tondello, Rina R Wehbe, Lisa Diamond, Marc Busch, Andrzej Marczewski, and Lennart E Nacke. 2016. The gamification user types hexad scale. In Proceedings of the 2016 annual symposium on computer-human interaction in play. 229-243.

[66] Juliane Weicker, Mareike Gabele, and Angelika Thöne-Otto. 2020. Motivation und Verhalten während eines selbstständig absolvierten Onlinetrainings. Abstract and Presentation: 35. Jahrestagung der Gesellschaft für Neuropsychologie 2020.

[67] Juliane Weicker, Nicole Hudl, Helmut Hildebrandt, Hellmuth Obrig, Magdalen Schwarzer, Arno Villringer, and Angelika Thöne-Otto. 2020. The effect of high vs. low intensity neuropsychological treatment on working memory in patients with acquired brain injury. Brain Injury (2020), 1-10.

[68] Juliane Weicker, Arno Villringer, and Angelika Thöne-Otto. 2016. Can impaired working memory functioning be improved by training? A meta-analysis with a special focus on brain injured patients. Neuropsychology 30, 2 (2016), 190.

[69] Nick Yee, Nicolas Ducheneaut, Les Nelson, and Peter Likarish. 2011. Introverted elves \& conscientious gnomes: the expression of personality in world of warcraft. In Proceedings of the SIGCHI conference on human factors in computing systems. 753-762.

[70] Chanuk Yoo, Mi-hyun Yong, Jaeyeop Chung, and Yeongae Yang. 2015. Effect of computerized cognitive rehabilitation program on cognitive function and activities of living in stroke patients. Journal of Physical Therapy Science 27, 8 (2015), 2487-2489.

\section{A RATINGS OF GAME ELEMENTS DEPENDING ON PLAYER TYPE IN PATIENTS}




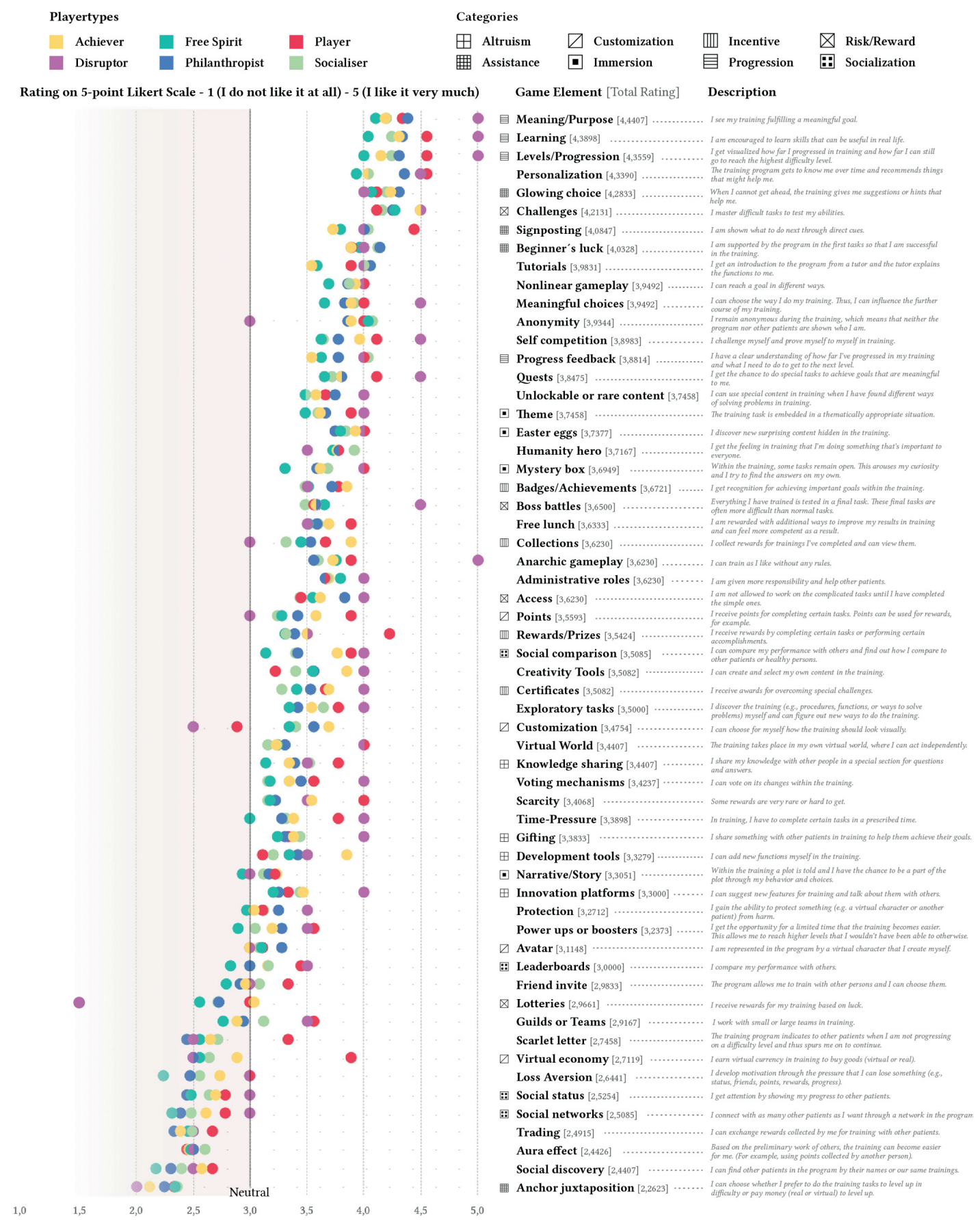

Figure 5: Ratings of Game Elements depending on Player Type in Patients 